\title{
Ode to oseltamivir and amantadine?
}

\author{
JM Conly MD ${ }^{1}, B L$ Johnston $M D^{2}$
}

$\mathrm{I}^{\mathrm{n}}$

nfluenza A and B viruses are the two major types of influenza Iviruses that cause human epidemic disease. Influenza A viruses are further categorized into subtypes based on two surface antigens: hemagglutinin $(\mathrm{H})$ and neuraminidase $(\mathrm{N})$. Influenza B viruses are not categorized into subtypes (1). Influenza A viruses are found in many animal species, including humans, ducks, chickens, pigs, whales, horses and seals, whereas influenza B viruses circulate only among humans. The $\mathrm{H}$ antigen contains common and strain-specific antigens, demonstrates antigenic variation, and acts as a site of attachment of the virus to host cells to initiate infection (1). The $\mathrm{N}$ antigen contains subtype-specific antigens and also demonstrates antigenic variation between subtypes. It is a surface glycoprotein possessing enzymatic activity essential for viral replication in both influenza $A$ and $B$ viruses. The $N$ antigen allows the release of newly produced virions from infected host cells, prevents the formation of viral aggregates after release from the host cells, and prevents viral inactivation by respiratory mucous $(2,3)$. It is thought that this enzyme may also promote viral penetration into respiratory epithelial cells and may contribute to the pathogenicity of the virus by promoting production of proinflammatory cytokines such as interleukin-1 and tumour necrosis factor from macrophages (4-6).

There are 16 different $\mathrm{H}$ subtypes and nine different $\mathrm{N}$ subtypes identified to date among influenza A viruses. Influenza A virus affecting humans has, in the past, traditionally involved three subtypes based on one of three $\mathrm{H}$ antigens ( $\mathrm{H} 1, \mathrm{H} 2$ and H3) and two additional subtypes based on one of two $\mathrm{N}$ antigens (N1 and N2). Influenza A viruses constantly change and adapt over time to infect and spread among humans. New influenza virus variants result from frequent antigenic change (ie, antigenic drift) resulting from point mutations that occur during viral replication. Influenza B viruses undergo this antigenic change less frequently than influenza $\mathrm{A}$ viruses. The term 'human influenza virus' usually refers to those subtypes that spread widely among humans.

Since 1977, influenza A virus subtypes H1N1 and H3N2, as well as influenza B viruses, have been in global circulation in the human population (1). In 2001, H1N2 viruses that likely emerged after genetic reassortment between human $\mathrm{H} 3 \mathrm{~N} 2$ and H1N1 strains began circulating widely (7). In addition, during the past decade, strains of influenza virus affecting humans, containing other $\mathrm{H}$ and $\mathrm{N}$ antigenic combinations, have been reported, including 'avian influenza' (8). Avian influenza is an infection caused by avian influenza viruses. Wild birds worldwide carry the viruses in their intestines but usually do not develop any illness. Wild birds are the primary natural reservoir for all subtypes of influenza A viruses and are thought to be the source of influenza A viruses in other animals. Most influenza viruses cause asymptomatic infection in wild fowl, but domesticated poultry, such as turkeys and chickens, can develop significant illness and die from avian influenza. Domesticated birds may become infected with avian influenza through direct contact with infected waterfowl or other infected poultry (which shed the virus in their saliva, nasal secretions and feces), or through contact with surfaces (such as dirt or cages) or materials (such as water or feed) that have been contaminated with the virus (8).

Avian influenza has been recognized for over a century. Although most virulent fowl influenza was caused by influenza A viruses containing the $\mathrm{H} 7$ antigen, $\mathrm{H} 5$ and other $\mathrm{H}$-containing subtypes have been associated with highly virulent influenza virus infections in fowl (9). Certain avian influenza A viruses, including some strains of $\mathrm{H} 5$ and $\mathrm{H} 7$ influenza viruses that cause widespread disease and death among fowl populations, would be considered highly pathogenic and may be associated with mortality rates of $90 \%$ to $100 \%$ in domestic fowl populations. Birds that survive influenza infection continue to shed virus via the fecal-oral route for at least 10 days, thus allowing further viral transmission by migratory birds or in crowded poultry markets where thousands of birds may be caged in close proximity.

The risk to humans from avian influenza has been considered low in the past, but confirmed cases of human infection from several subtypes of avian influenza infection have been reported since 1997. Most cases of avian influenza infection in humans have resulted from contact with infected poultry (eg, domesticated chicken, ducks and turkeys) or surfaces contaminated with secretions or excretions from infected birds. Avian influenza strains affecting humans were first reported from Hong Kong during the 1997 influenza season and were shown to be derived from avian sources (10-12). This first avian influenza A virus (H5N1) affected 18 humans during the $1997 / 1998$ influenza season and was associated with six deaths. A case-control study identified recent exposure to live poultry as an important risk factor for infection with this avian strain infection (13), and cohort studies have suggested that humanto-human transmission of the H5N1 virus was limited $(14,15)$.

\footnotetext{
${ }^{1}$ Departments of Pathology and Laboratory Medicine, Medicine, and Microbiology and Infectious Diseases, Centre for Antimicrobial Resistance,

University of Calgary, Calgary, Alberta; ${ }^{2}$ Queen Elizabeth II Health Sciences Centre and Dalhousie University, Halifax, Nova Scotia

Correspondence: Dr JM Conly, Foothills Medical Centre, 1403-29 Street South, Calgary, Alberta T2N 2T9. Telephone 403-944-8222,

fax 403-944-1095, e-mail jconly@ucalgary.ca

Received and accepted for publication January 30, 2006
} 
Additional cases of confirmed H5N1 influenza occurred in Hong Kong in February 2003, causing two deaths (1). From late 2003 to February 2004, outbreaks of H5N1 infection in poultry were detected in several Asian countries, notably Vietnam, Thailand, Cambodia, China, Indonesia, Japan, Laos and the Republic of Korea (16). At that time, more than 100 million birds in the affected countries either died from the disease or were killed in an attempt to control the outbreaks. By March 2004, the outbreak was reported to be under control. However, since June 2004, new outbreaks of the H5N1 strain among poultry have been reported by a number of countries in Asia (Cambodia, China, Tibet, Indonesia, Kazakhstan, Malaysia, Mongolia, Russia, Thailand and Vietnam). H5N1 infection has also been reported among poultry in Turkey, Romania and Ukraine (17). Outbreaks of the H5N1 virus have been reported among wild migratory birds in China, Croatia, Mongolia and Romania. Between 2004 and early 2005, a total of 89 laboratory-confirmed human infections, 52 of which were fatal, were reported to the World Health Organization by public health authorities in Vietnam, Thailand and Cambodia (18). In addition, as of January 2006, human cases of H5N1 infection have been reported in Cambodia, China, Indonesia, Thailand, Vietnam and, most recently, Turkey.

Although the mainstay of influenza prevention and control remains annual vaccination, the adjunctive use of antiviral drugs also plays a role, given the continued presence of new human influenza strains and the new threat posed by emerging avian influenza strains (7). Two classes of antiviral medications are currently available and include the adamantanes (amantadine and rimantadine) and the $\mathrm{N}$ inhibitors (oseltamivir and zanamivir) $(19,20)$. Amantadine and rimantadine have activity against only influenza $\mathrm{A}$ strains and are used for both treatment and chemoprophylaxis of influenza $\mathrm{A}$. The $\mathrm{N}$ inhibitors are active against both influenza $\mathrm{A}$ and $\mathrm{B}$ viruses. A recent report from the Centers for Disease Control and Prevention (CDC) (21) describes significant resistance of influenza A viruses currently circulating in the United States (US) to amantadine and rimantadine, and recommends against the use of these drugs for the remainder of the 2005/2006 influenza season.

Resistance of influenza A viruses to amantadine and rimantadine can occur spontaneously or emerge rapidly during treatment (22), usually via single point mutations in the codons for amino acids (23). It is noteworthy that neither transmission nor virulence of amantadine-resistant influenza $\mathrm{A}$ viruses are impaired (24). In the US, resistance to amantadine and rimantadine increased from 1.9\% during the 2003/2004 influenza season to $11 \%$ during the $2004 / 2005$ season. From October 1, 2005, to January 14, 2006, the CDC tested 123 influenza A viruses, collected from 23 states, for resistance to amantadine and rimantadine. Of the $120 \mathrm{H} 3 \mathrm{~N} 2$ influenza A strains tested, 109 (91\%) had resistance to amantadine and rimantadine. Of the H1N1 viruses tested, none had any mutations associated with resistance. All of the US influenza viruses tested for antiviral resistance were susceptible to $\mathrm{N}$ inhibitors. On the basis of available antiviral testing results, the CDC recommended against the use of amantadine and rimantadine for the treatment or chemoprophylaxis of influenza A infections in the US for the remainder of the 2005/2006 influenza season.

The Public Health Agency of Canada's National Microbiology Laboratory recently tested 47 H3N2 isolates and found that $43(91 \%)$ were resistant to amantadine. The samples tested were collected from four provinces (British Columbia, Ontario, Saskatchewan and Alberta) during the current 2005/2006 influenza season (24). The results, similar to those from the US, represented a significant increase in resistance compared with previous years and were consistent with the level of antiviral resistance recently reported by the CDC. It is recommended that either oseltamivir or zanamivir be prescribed if an antiviral medication is indicated for the treatment of influenza, and that oseltamivir be prescribed for chemoprophylaxis of influenza.

Although the use of antivirals is recommended by some for the control of seasonal and pandemic influenza, a recently published systematic review by Jefferson et al (25) examined the evidence for the efficacy, effectiveness and safety of registered antivirals against influenza in healthy adults. Their findings cast doubt on the utility of both the adamantanes and the $\mathrm{N}$ inhibitors (25). Those investigators searched various databases to the end of 2005, contacted manufacturers, corresponded with authors and identified 54 randomized, controlled trials comparing prophylactic $(\mathrm{n}=27)$ or treatment $(n=27)$ efficacy against symptomatic or asymptomatic influenza, expressing prophylactic efficacy as a proportion (1-relative risk [RR]) in their meta-analysis. An analysis of 51 reports of 52 randomized, controlled trials revealed that amantadine prevented $61 \%$ (95\% CI 35 to 76 ) of influenza A cases and 25\% (95\% CI 13 to 36) of cases of influenza-like illness. However, amantadine was associated with nausea (OR 2.56; 95\% CI 1.37 to 4.79), insomnia and hallucinations (OR 2.54; 95\% CI 1.50 to 4.31), and withdrawals (OR 2.54; 95\% CI 1.60 to 4.06$)$ due to adverse events. In addition, amantadine had no effect on asymptomatic cases (RR 0.85; 95\% CI 0.40 to 1.80 ). In treatment trials, amantadine significantly shortened the duration of fever compared with placebo (by 0.99 days, $95 \%$ CI -1.26 to -0.71 ), but it did not affect nasal shedding of influenza A viruses (RR 0.93; 95\% CI 0.71 to 1.21). There were fewer data for rimantadine, but its effects were comparable with those of amantadine.

For prophylaxis, $\mathrm{N}$ inhibitors had no effect compared with placebo against influenza-like illness using standard doses of oseltamivir (75 mg once daily) (RR 1.28 ; $95 \%$ CI 0.45 to 3.66 ) and zanamivir (10 mg once daily via inhalation) (RR 1.51; $95 \%$ CI 0.77 to 2.95), respectively. For symptomatic influenza, the efficacy of oral oseltamivir was $61 \%$ (95\% CI 15 to 82$)$ at $75 \mathrm{mg}$ once daily and $73 \%$ (95\% CI 33 to 89 ) at $150 \mathrm{mg}$ daily. Inhaled zanamivir (10 mg once daily) had an efficacy of $62 \%$ (95\% CI 15 to 83 ), but neither $\mathrm{N}$ inhibitor was effective against asymptomatic influenza. Oseltamivir was associated with nausea (OR 1.79; 95\% CI 1.10 to 2.93), which was greater especially at higher prophylactic doses (OR 2.29; 95\% CI 1.34 to 3.92). For postexposure prophylaxis, oseltamivir had a protective efficacy of 58.5\% (95\% CI 15.6 to 79.6 ) for households and from 68\% (95\% CI 34.9 to 84.2 ) to $89 \%$ (95\% CI 67 to 97) for contacts of index cases. Compared with placebo, the hazard ratios for time to alleviation of influenza symptoms were 1.33 (95\% CI 1.29 to 1.37 ) for zanamivir and 1.30 (95\% CI 1.13 to 1.50$)$ for oseltamivir when the medication was started within $48 \mathrm{~h}$ of symptom onset. Both drugs significantly reduced viral nasal titres (weighted mean difference $-0.62 ; 95 \% \mathrm{CI}-0.82$ to -0.41 ). In influenza cases, oseltamivir at $150 \mathrm{mg}$ daily was effective in preventing lower respiratory tract complications (OR 0.32; $95 \%$ CI 0.18 to 0.57 ). No credible data on the effects of oseltamivir on avian influenza could be found (25). 
Jefferson et al suggested that the evidence does not support the use of adamantanes for influenza and that $\mathrm{N}$ inhibitors should not be used routinely for seasonal influenza, but only with associated public health measures in a pandemic situation. Their findings suggested that amantadine and rimantadine have an effect on the symptoms of influenza A but do not prevent infection and do not affect viral shedding. Because of the potential for serious adverse events and rapid onset of antiviral resistance, they also indicated that the use of amantadine and rimantadine should be discouraged for seasonal and pandemic influenza, especially if used in isolation from other measures. Given the current levels of antiviral resistance to the adamantanes of the H3N2 strains of influenza A presently circulating in North America, these findings are a sobering thought as the medical community engages in pandemic planning for influenza.

In addition, their findings indicated that the $\mathrm{N}$ inhibitors reduced influenza symptoms by either preventing their appearance or curtailing their duration. In addition, there was clear evidence of the ability of $\mathrm{N}$ inhibitors to interrupt transmission of seasonal influenza in households, but they did not appear to prevent asymptomatic infection and did not interrupt nasal shedding of seasonal influenza viruses. Jefferson et al suggested that the prophylactic use of $\mathrm{N}$ inhibitors in a serious epidemic or a pandemic could enhance vulnerability to infection by preventing seroconversion and facilitate the selection of mutant viruses resistant to $\mathrm{N}$ inhibitors (25). This latter point has serious implications for the use of these agents in a pandemic of avian influenza. Other evidence is accumulating that raises cautionary notes about the $\mathrm{N}$ inhibitors and their potential efficacy and resistance development. Oseltamivir has been used against three subtypes of avian influenza viruses with proven bird-to-human and human-to-human transmission: H5N1, H7N7 and H7N3. Yuen and Wong (26) reviewed the experience from the avian influenza cases in southeast Asia, which suggested that viral shedding commences before symptoms appear and ceases $48 \mathrm{~h}$ after symptom onset. Additional data from $\mathrm{H} 5 \mathrm{~N} 1$ influenza cases suggest that viral shedding and infectivity of index cases can be protracted and viral load may be up to 10 times greater than in seasonal influenza (8). In these outbreaks in southeast Asia, the use of oseltamivir did not appear to have any impact on mortality, although other factors, such as late initiation of therapy and high initial viral load, may have been confounding variables. Resistance to oseltamivir was detected in up to $16 \%$ of children given the drug (8) and, in a recent report (27), was detected in two of eight Vietnamese persons aged eight to 35 years (27). In one of the patients in the latter study (27), the development of resistance while on therapy with oseltamivir coincided with clinical deterioration and death, as well as the appearance of increasing viral load in the throat during the clinical deterioration. This report (27) also corroborated earlier findings about incomplete viral suppression. Resistant viral isolates have also been detected in up to $18 \%$ of children who have received oseltamivir for H1N1 or H3N2 infections (28-30). The apparently common feature favouring the selection of resistant viruses appears to be immunological naivety to the infecting viral subtype.

With avian influenza harbouring potentially greater virulence and higher viral load burden than those of seasonal human influenza viruses, the use of $\mathrm{N}$ inhibitors in a serious epidemic or pandemic may not hold as much promise as originally thought. Jefferson et al suggest that the inability of
$\mathrm{N}$ inhibitors to prevent infection and suppress viral nasal excretion raises doubts as to their effectiveness in interrupting viral spread (25). They also suggest that overestimation of the capacity of antivirals to prevent illness could alter the behaviour of recipients and favour viral spread. Collectively, these recent findings of incomplete viral suppression with high viral burden in avian influenza and rapid resistance development in both human and avian influenza A to antivirals raise serious questions about the stockpiling of antiviral agents and the emphasis on the use of $\mathrm{N}$ inhibitors as a major component of the armamentarium for an emerging pandemic. The need for continued study into influenza and its pharmacotherapy cannot be overemphasized.

\section{REFERENCES}

1. Nicholson KG, Wood JM, Zambon M. Influenza. Lancet 2003;362:1733-45

2. Liu C, Air GM. Selection and characterization of a neuraminidaseminus mutant of influenza virus and its rescue by cloned neuraminidase genes. Virology 1993;194:403-7.

3. Buck P, Herman S, Scott C, et al. Respiratory virus surveillance. FluWatch project, 1997-1998. Can Commun Dis Rep 1998;24:49-51.

4. Colman PM. Neuraminidase: Enzyme and antigen. In: Krug RM, ed. The Influenza Viruses. New York: Plenum Press, 1989:175-218.

5. Colman PM. Influenza virus neuraminidase: Structure, antibodies, and inhibitors. Protein Sci 1994;3:1687-96.

6. Schultz-Cherry S, Hinshaw VS. Influenza virus neuraminidase activates latent transforming growth factor beta. J Virol 1996;70:8624-29.

7. Harper SA, Fukuda K, Uyeki TM, Cox NJ, Bridges CB; Advisory Committee on Immunization Practices (ACIP), Centers for Disease Control and Prevention (CDC). Prevention and control of influenza. Recommendations of the Advisory Committee on Immunization Practices (ACIP). MMWR Recomm Rep 2005;54(RR-8):1-40. (Erratum in 2005;54:750).

8. Beigel JH, Farrar J, Han AM, et al; Writing Committee of the World Health Organization (WHO) Consultation on Human Influenza A/H5. Avian influenza A (H5N1) infection in humans. N Engl J Med 2005;353:1374-85.

9. Horimoto T, Kawaoka Y. Pandemic threat posed by avian influenza A viruses. Clin Microbiol Rev 2001;14:129-49.

10. Centers for Disease Control and Prevention (CDC). Update: Isolation of avian influenza $\mathrm{A}(\mathrm{H} 5 \mathrm{~N} 1)$ viruses from humans Hong Kong, 1997-1998. MMWR Morb Mortal Wkly Rep 1998;46:1245-7.

11. Claas EC, Osterhaus AD, van Beek R, et al. Human influenza A $\mathrm{H} 5 \mathrm{~N} 1$ virus related to a highly pathogenic avian influenza virus. Lancet 1998;351:472-7. (Erratum in 1998;351:1292).

12. Lee SY, Mak KH, Saw TA. The avian flu (H5N1): One year on. Department of Health, Hong Kong Special Administrative Region of China. Public Health and Epidemiology Bulletin 1999;8:1-7.

13. Mounts AW, Kwong H, Izurieta HS, et al. Case-control study of risk factors for avian influenza A (H5N1) disease, Hong Kong, 1997. J Infect Dis 1999;180:505-8.

14. Buxton Bridges C, Katz JM, Seto WH, et al. Risk of influenza A (H5N1) infection among health care workers exposed to patients with influenza A (H5N1), Hong Kong. J Infect Dis 2000;181:344-8.

15. Katz JM, Lim W, Bridges CB, et al. Antibody response in individuals infected with avian influenza A (H5N1) viruses and detection of anti-H5 antibody among household and social contacts. J Infect Dis 1999;180:1763-70.

16. World Health Organization (WHO). Avian influenza A(H5N1) update 32: Situation (human) in Thailand. <www.who.int/csr/don/ 2004_03_09/en> (Version current at February 1, 2006).

17. Centers for Disease Control and Prevention. Key facts about avian influenza (bird flu) and avian influenza A (H5N1) virus $<$ www.cdc.gov/flu/avian/gen-info/facts.htm > (Version current at February 1, 2006).

18. World Health Organization. Cumulative Number of Confirmed Human Cases of Avian Influenza A(H5N1) since 28 January 2004. $<$ www.who.int/csr/disease/avian_influenza/country/ cases_table_2005_01_26/en> (Version current at February 1, 2006) 
19. Aoki F. Amantadine and rimantadine. In: KG Nicholson, RG Webster, AJ Hay, eds. Textbook of influenza. Oxford: Blackwell Science, 1998:457-76.

20. Moscona A. Neuraminidase inhibitors for influenza. N Engl J Med 2005;353:1363-73.

21. Centers for Disease Control and Prevention (CDC). High levels of adamantane resistance among influenza A (H3N2) viruses and interim guidelines for use of antiviral agents - United States, 2005-06 influenza season. MMWR Morb Mortal Wkly Rep 2006;55:44-6.

22. Belshe RB, Smith MH, Hall CB, Betts R, Hay AJ. Genetic basis of resistance to rimantadine emerging during treatment of influenza virus infection. J Virol 1988;62:1508-12.

23. Bright RA, Medina MJ, Xu X, et al. Incidence of adamantane resistance among influenza A (H3N2) viruses isolated worldwide from 1994 to 2005: A cause for concern. Lancet 2005;366:1175-81.

24. Public Health Agency of Canada. Interim recommendation for use of amantadine for influenza. <www.phac-aspc.gc.ca/media/ advisories_avis/2006/statment060115.html > (Version current at February 3, 2006).

25. Jefferson T, Demicheli V, Rivetti D, Jones M, Di Pietrantonj C, Rivetti A. Antivirals for influenza in healthy adults: Systematic review. Lancet 2006;367:303-13.

26. Yuen KY, Wong SS. Human infection by avian influenza A H5N1. Hong Kong Med J 2005;11:189-99.

27. de Jong MD, Tran TT, Truong HK, et al. Oseltamivir resistance during treatment of influenza A (H5N1) infection. N Engl J Med 2005;353:2667-72.

28. Whitley RJ, Hayden FG, Reisinger KS, et al. Oral oseltamivir treatment of influenza in children. Pediatr Infect Dis J 2001;20:127-133. (Erratum in 2001;20:421).

29. Kiso M, Mitamura K, Sakai-Tagawa Y, et al. Resistant influenza A viruses in children treated with oseltamivir: Descriptive study. Lancet 2004;364:759-65.

30. Ward P, Small I, Smith J, Suter P, Dutkowski R. Oseltamivir (Tamiflu) and its potential for use in the event of an influenza pandemic. J Antimicrob Chemother 2005;55(Suppl 1):i5-i21. 


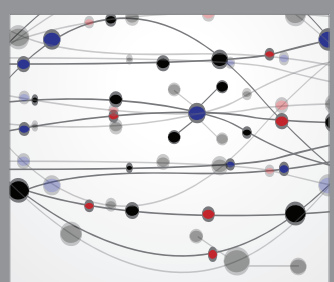

The Scientific World Journal
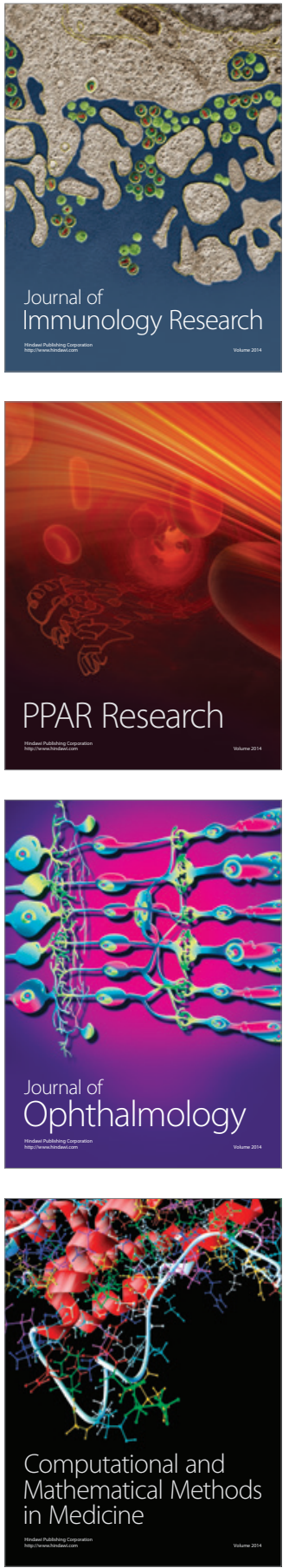

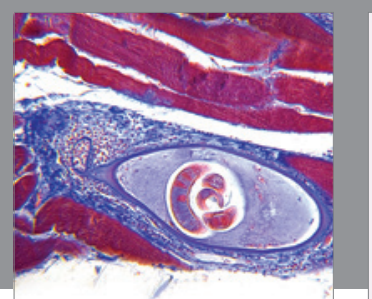

Gastroenterology Research and Practice

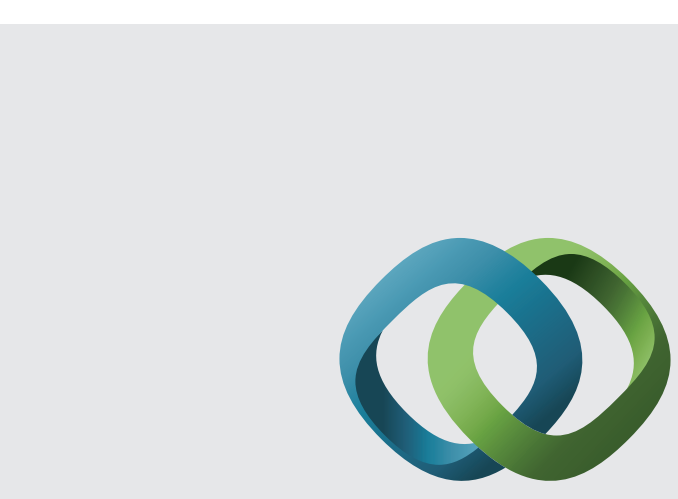

\section{Hindawi}

Submit your manuscripts at

http://www.hindawi.com
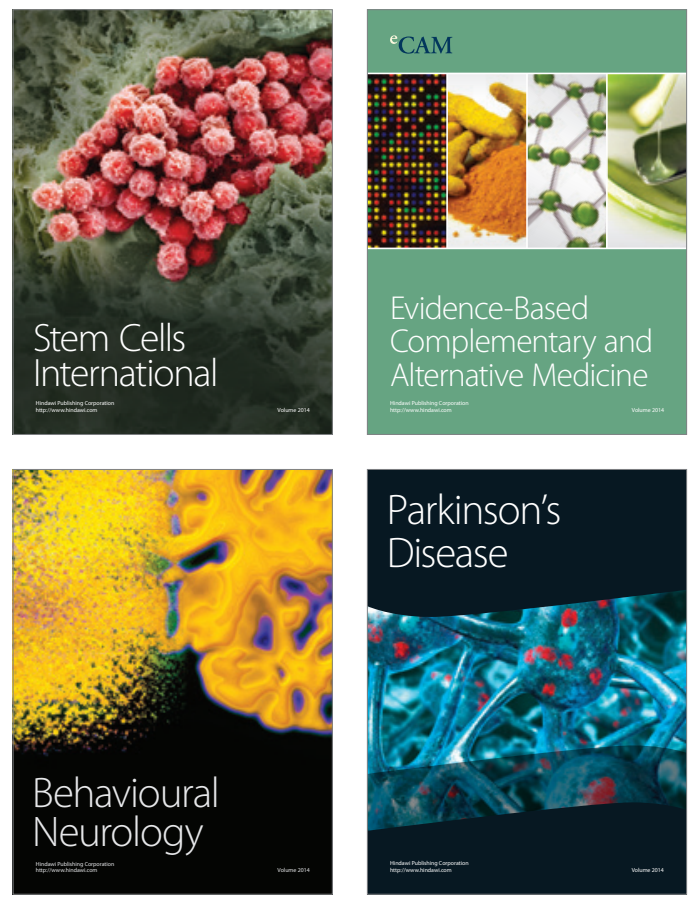
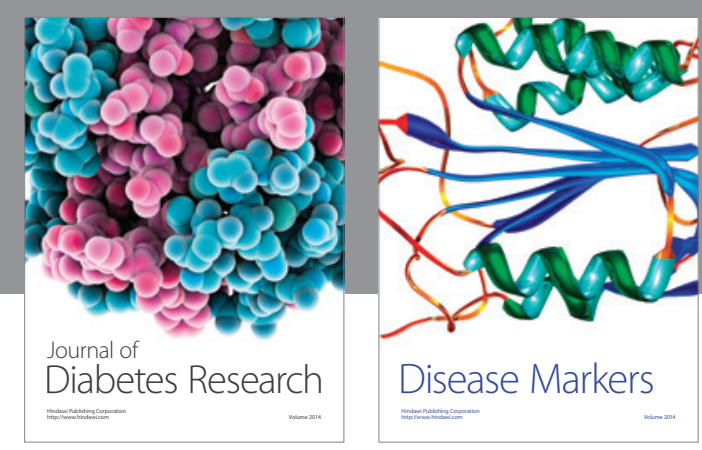

Disease Markers
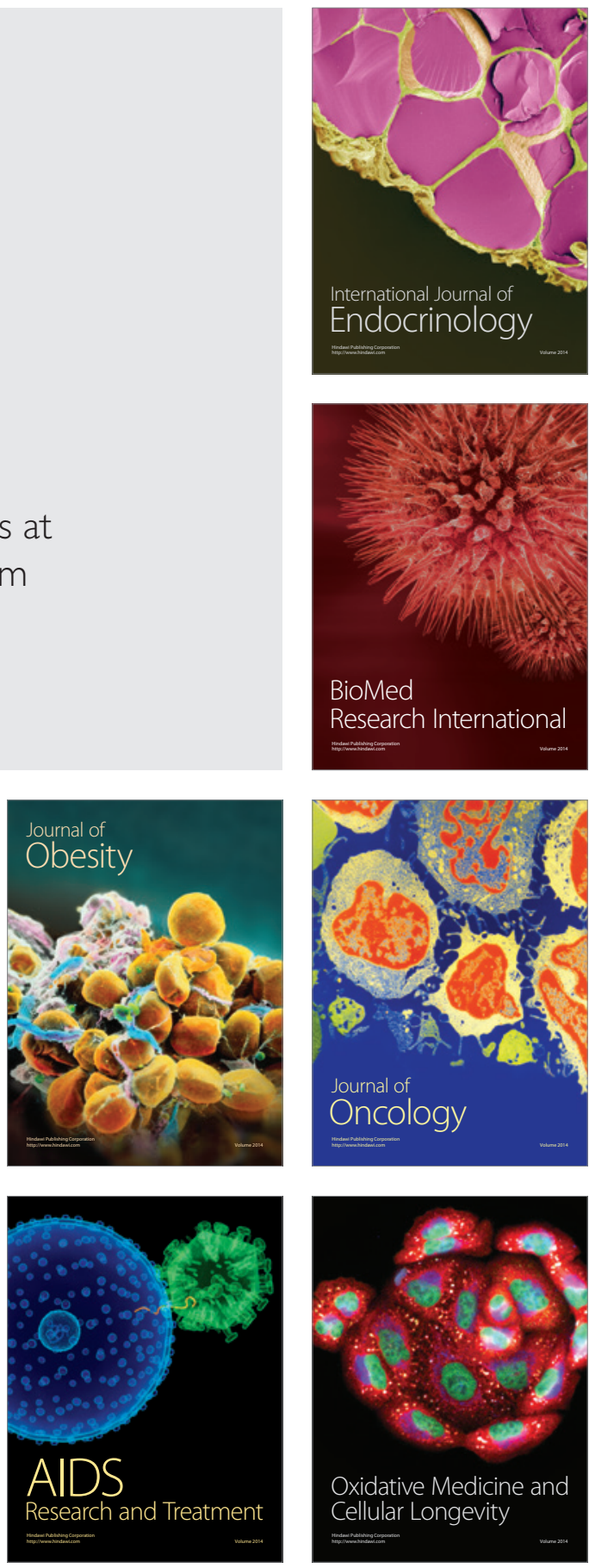